\title{
An Approach to Improve Palmprint Recognition Accuracy by using Different Region of Interest Methods with Local Binary Pattern Technique
}

\author{
Mouad M.H. Ali ${ }^{*}$, A.T. Gaikwad ${ }^{2}$ and Pravin Yannawar ${ }^{1}$ \\ 'Department of CS\&IT, Vision and Intelligent System Lab, Dr. Babasaheb Ambedkar Marathwada University, \\ Aurangabad - 431001, Maharashtra, India; \\ Mouad198080@gmail.com, pravinyannawarr@gmail.com, \\ ${ }^{2}$ Institute of Management Studies and Information Technology, Aurangabad - 431001, Maharashtra, India; \\ drashoktgaikwad@gmail.com
}

\begin{abstract}
Objective: To extract the Region of Interest (ROI) of palmprint image by using appropriate methods and to improve the accuracy of palmprint recognition system. Methods/Statistical Analysis: This piece of work is primarily addressing the different mechanisms for extracting ROI area. The techniques like Competitive Hand Valley Detection (CHVD), and Euclidean Distance (ED) were applied as the part of pre-processing, while the Feature Extraction mechanism LBP was utilized to extract the texture feature from different type of ROIs of palmprint image. Findings: The experimental results showed that CHVD with LBP gave best result with high accuracy reached to $96.10534 \%$ and Equal Error Rate (EER) of 3.894661\%, while in ED the best result showed accuracy reached to $88.23611 \%$ and EER of $11.76389 \%$. Application/Improvements: The study mainly concentrated on developing palmprint authentication system with less EER and high accuracy.
\end{abstract}

Keywords: Analysis, Competitive Hand Valley Detection (CHVD), Euclidean Distance, Local Binary Pattern (LBP), Palmprint Recognition

\section{Introduction}

Today, personal identification playsan active research and applications in our daily life like banking, immigration, and access control, ID card, passport office and country borders etc. Recently, the biometric application (identification or verification) has widely used and majority of research studies have conducted in the field of security system with different technologies (modalities or traits) such as fingerprint, iris, palmprint, face, retina etc ${ }^{1}$. Biometric is the automatic system which is used to recognize the persons by their characterizations (behavioral and physical) ${ }^{2}$. The main objective of any biometric system is to achieve low cost, less error rate, speed in performance and high accuracy.
There are two types of palmprint which called high resolution and low resolution. Each type is suitable for different applications. High resolution images are used for forensicapplication ${ }^{3}$ while low resolution images are used for access controls application.

Palmprint trait has a rich of information which is useful for identification and verification propose. Different types of feature can be extracted from high and low resolution palmprint images. The features from high resolution images include Minutiae feature, Ridges features and Singular point feature ${ }^{4-5}$. Whereas, the features from low resolution images include Principal line, Texture feature, Palm-crease, Wrinkles and Statistical features. All these features present on the surface of palmprint images.

${ }^{*}$ Author for correspondence 
The methods of palmprint can be classified into different classes depending upon verification and identification. The methods used in verification system include Line based methods, subspace based methods and statistical based methods. Line-based methods are focused on edge detectors to extract the palm lines from the palmprint images such as mention in $\frac{6-14}{\text {. }}$ Matching of these lines can be made either directly or indirectly by using different matching format. Subspace based methods, PCA method, LDA method and ICA method were studied in earlier works $\frac{15-18}{18}$ other methods like wavelets, Gabor, DCT and Kernels were mentioned in $\frac{19-22}{}$.

Statistical methods are either local or global. In the case of Local the images should be transformed to another domain and then divided into several small regions $\frac{23-27}{}$. The means, standard divisions and variances of each region were calculated and stored as features. Some of researchers used Gabor, wavelets and Fourier transforms ${ }^{28-30}$. Another researcher used Local Binary Pattern (LBP) histograms as features ${ }^{31}$. Whereas, some of them used global statistical methods like Moments, centers of gravity and density as mention in ${ }^{31-36}$.

The remaining sections of the paper are discussed the methodology system which includes the preprocessing, ROIs methods, feature extraction, matching and decision that covered in section 2. The results and discussion are given in details in section 3. Section 4 exhibits the conclusion and future work.

\section{Methodology of the System}

In this section the methodology of the palmprint recognition system is discussed. The ROI from palmprint images was extracted by two ways called as CHVD, and ED. The ROI was passed to feature extraction techniques to extract the texture feature by using LBP technique, and then the feature reduction technique was utilized to reduce the dimensionality by PCA method. After that the features were stored as data template which used for matching purposes. This section was divided into different subsections. Section 2.1 which covers the ROI methods in details, section 2.2 which discusses LBP feature extraction methods, section 2.3 which represents the matching process and section 2.4 which shows the decision. Figure 1 shows the block diagram of methodology of plamprint recognition system.

\subsection{ROI Extraction Methods}

The ROI refers to a subset of all the data used in an observation. In palmprint ROI is the data of two- dimensional and defines as rectangular area on the hand surface. It is a small area which includes more information like Minutiae, Principal line, and the key point. The key points involve an end points or a branch points ${ }^{37}$ which needed in the process of identification or verification. There are many techniques utilized to extract the ROI from palm image such as CHVD and ED. There are two general steps performed before making ROI algorithm

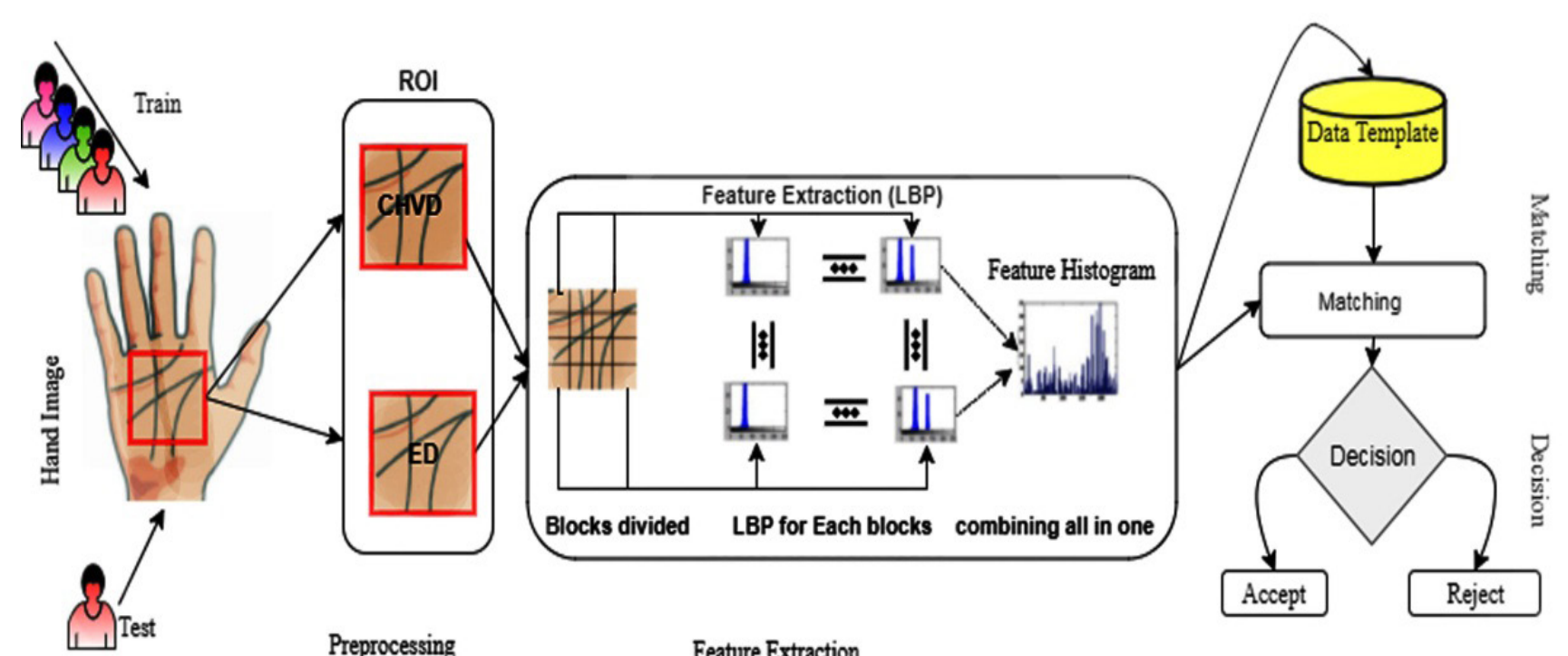

Figure 1. Methodology system of palmprint recognition system. 
namely: image Binarization and boundary extraction which include in the pre-processing stage as shown in (Figure 2).

Image Binarization: It is the process of converting gray scale images into black-and-white image based on threshold value by multiplying a coefficient $\alpha$ with the greatest possible values of the scale of gray used $(\theta=\alpha * 2 n-1)$ where $\mathrm{n}$ is a fine gray scale used and $\theta$ is the threshold value (T). The value is changed for each pixel in image by Eq. (1) where $x$ is the pixel values at particular coordinates. 0 represents black and 1 represents white.

$$
f(x)=\left\{\begin{array}{l}
0 ; x<\theta \\
1 ; x \geq \theta
\end{array}\right.
$$

Boundary Extraction: Boundary objects can be generated from the black-and-white image by applying erosion operator on the matrix structuring element. The boundary of palmprint image can be obtained by Eq. (2):

Boundary $(B W$ image $)=B W$ image $-(B W$ image $\Theta S)(2)$
Where $\theta$ is an operator to perform erosion on a black and white image and $S$ is the structuring element matrix which can be represented as shown in Eq. (3).

$$
S=\left[\begin{array}{lll}
0 & 1 & 0 \\
1 & 1 & 1 \\
0 & 1 & 0
\end{array}\right]
$$

\subsubsection{Competitive Hand Valley Detection (CHVD)}

The CHVD algorithm was used to extract the ROI area from palm image based on four reference points which called the valley points that obtained by tracking the boundary and seeking the coordinates of all the points. The valley point is a pixel where most of its neighboring pixels are in the object area of palmprint. Figure 3 represents a block diagram of extracting the ROI using CHVD technique.

A CHVD algorithm checks whether a pixel included a valley point by different cases as discusses below:

Case 1: Putting four points test with same distance from pixel (current pixel) that will be checked whether

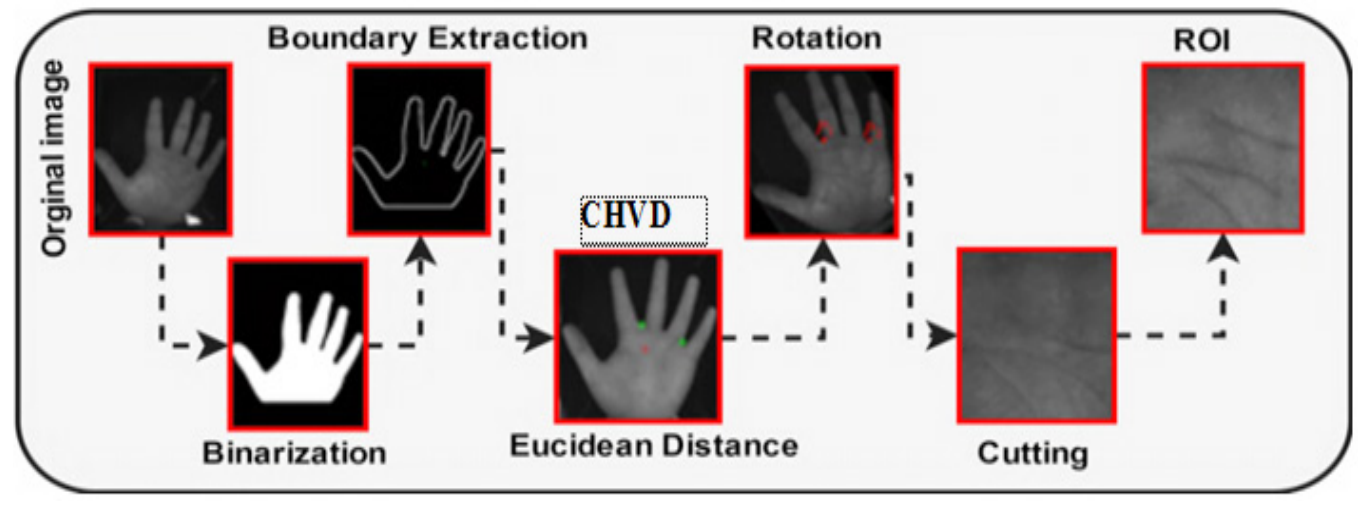

Figure 2. The main steps of palmprint pre-processing.

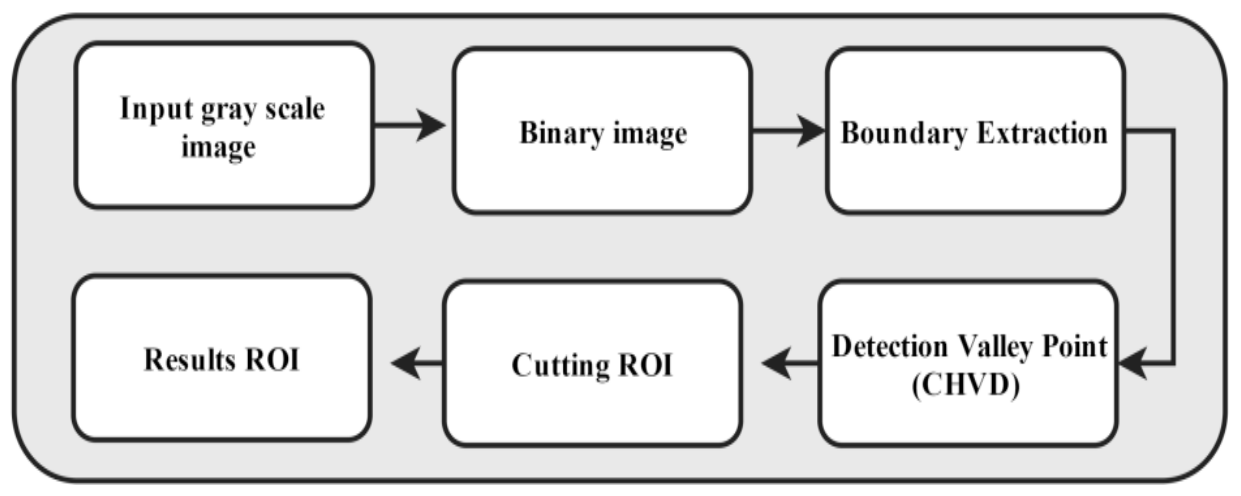

Figure 3. Block diagram of CHVD algorithm. 
the pixel is a valley point. If one point is in the background while other areas are in the area then the current pixel palmprint be a candidate as a valley point and then do check the current pixel to the next condition, if not then the checks carried out for the next pixel,

Case 2: Test point increase to eight by the distance from the current $\beta+\alpha$ pixel. Make checks if at least one fruit or not more than four test points are in the background area and the rest are in the area palmprint the current pixel still be a candidate valley point and go to the next check,

Case 3: Test point increased to 16 with a distance $\beta+\alpha+\mu$ pixel (current pixel). If at least one fruit or not more than seven points are located on the background area and the rest are in the area palmprint the current pixel still be a candidate valley point and continued to check the latest condition, and

Case 4: Pull a straight line from the current pixel to the area of non-palmprint. If the line does not intersect with the current point palmprint area is considered as the valley point.

CHVD algorithm is based on the assumption that no person flexing his fingers over $120^{\circ} \underline{38}$. The main problem in the CHVD algorithm is certain conditions can produce more than one candidate valley point. The solution is to select a point depend on the $y$ axis value of the greatest or is at the bottom of the image of the four valley point $\left\{\mathrm{P}_{1}, \mathrm{P}_{2}, \mathrm{P}_{3}, \mathrm{P}_{4}\right\}$ two points taken as a reference point that is $\mathrm{P}_{1}$ and $\mathrm{P}_{2}$ as shown in Figure 4. ROI generation differs in terms of size and orientation. Then, the next step is to use the amount of the angle rotates ROI obtained from two reference points and changing all the ROI into a standard size using the Bicubic Interpolation. Figure 5 shows our developing of the Graphical User Interface (GUI) of automatic system for extracting ROI by using CHVD method.

\subsubsection{Euclidean Distance}

The idea of taking ROI using Euclidean Distance method is the same as CHVD technique. However, they differ by the way of getting the reference point. Figure 6 shows the block diagram of the ROI retrieval process by using Euclidean Distance.
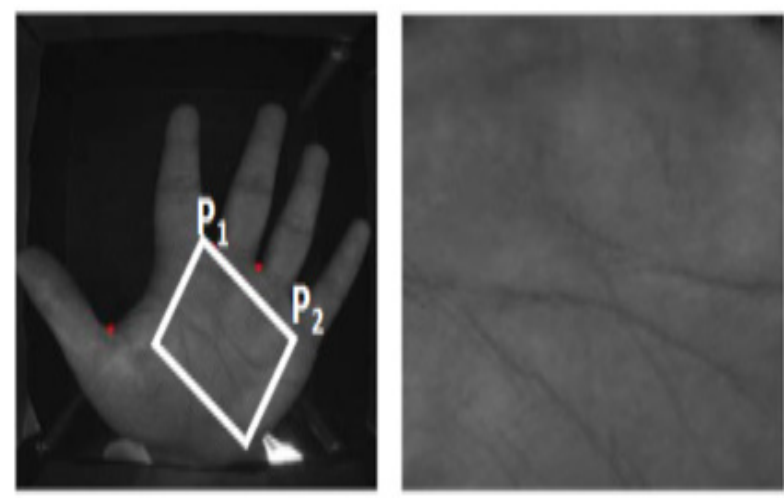

Figure 4. Making ROI process by CHVD.

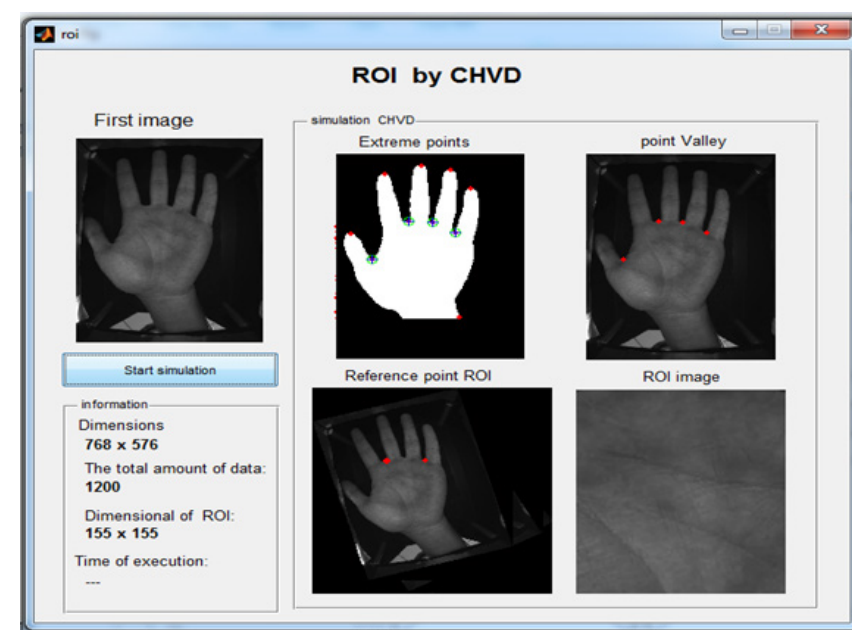

Figure 5. GUI of automatic ROI system by CHVD algorithm.

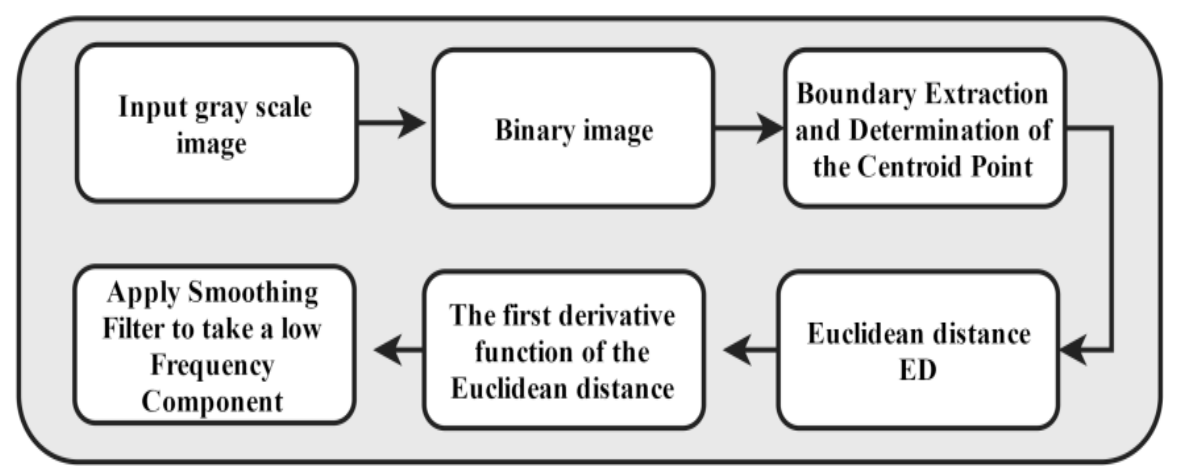

Figure 6. Block diagram of Euclidean distance. 
The way to determine the centroid point of an object in a black-and-white image was to find the mean values of $x$ and $y$ of the overall coordinates point which is the location of the pixel that represents the object. The centroid coordinate point can be searched using Eq. (4).

$$
x_{\text {centroid }}=\frac{1}{N} \sum_{i=1}^{N} x_{i} \text { and } y_{\text {centroid }}=\frac{1}{N} \sum_{i=1}^{N} y_{i}
$$

Where $x_{i}$ and $y_{i}$ are the values of $x$ and $y$ at $\mathrm{p}(x, y)=1$.

The next step was to calculate the distance between each point on the boundary with its centroid point using the Eq. (5).

$$
\operatorname{Dist}(x, y)=\sqrt{\left(x-x_{\text {centroid }}\right)^{2}+\left(y-y_{\text {centroid }}\right)^{2}}
$$

All the distance values resulting from Euclidean distance were calculated and plotted as shown in the Figure 7 a.

The peak point shows the pixel location of the fingertips while the valley point indicates the pixel location of the valley point seen from its distance to the centroid point. To obtain the coordinates of the valley point, first find the first derivative of the Euclidean function and then mark the dots passing the zero on the y-axis (zero-crossing points). Figure $7 \mathrm{~b}$ shows the first derivative value of the Euclidean function.

Both the vertex and valley points passed through the value 0 on the $y$-axis. However, in the graph above a lot of zero-crossing caused by the erratic contours of the palmprint image ${ }^{39}$ so that the valley point location cannot be determined yet. This can be overcome by smoothing the graph by removing high frequency components and maintaining low frequency components. Figure $7 \mathrm{c}$ shows the results of the smoothing.

Valley point can be identified from the point that passed the value 0 on the $y$-axis and changed the sign from negative to positive. The reference point used is only the valley point between the index finger and the middle finger $\left(\mathrm{V}_{1}\right)$ and between the ring finger and the little finger $\left(\mathrm{V}_{3}\right)$ as shown in Figure 8.

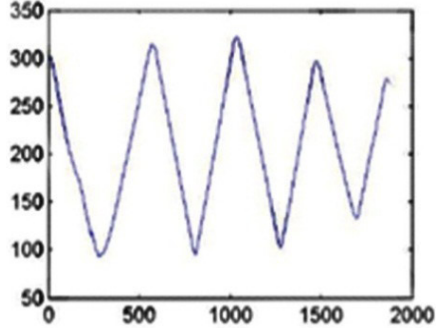

(a)

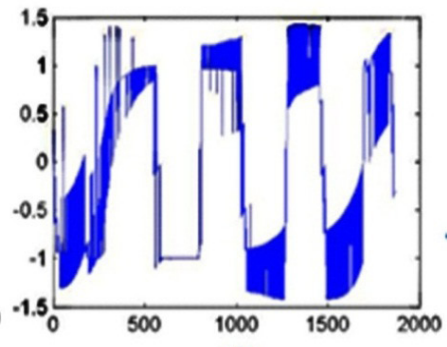

(b)

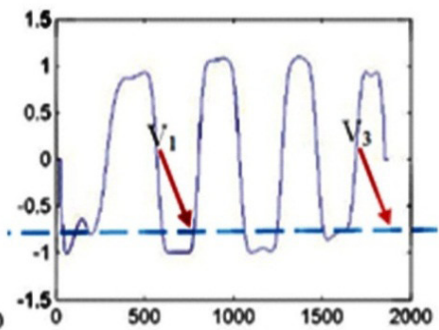

(c)

Figure 7. Extract ROI Method by ED: (a) Euclidean distance of each pixel within the boundary with the centroid, (b) First derivative value, and (c) smoothing value of First derivative graph.

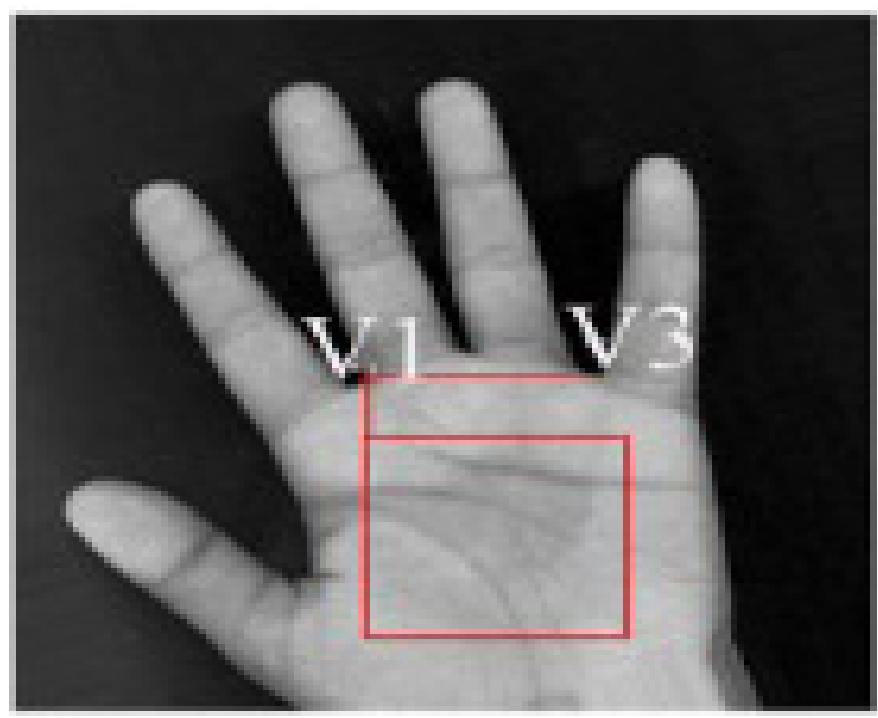

Figure 8. ROI detection of a palm image by ED. 
Then point $\mathrm{V}_{1}$ and $\mathrm{V}_{3}$ were used to find the angle of rotation so that the ROI is the same for the hand with different orientations. The final step was to draw a straight line from point $\mathrm{V}_{1}$ to point $\mathrm{V}_{3}$ to form the ROI area. Figure 9 shows our developing system of extracting the ROI by Euclidean Distance method.

\subsection{Feature Extraction using LBP}

LBP operator considered as a texture feature and it is used for shape extraction of gray scale image. It refers to a binary code for an image-pixel and it provides us by information regarding the local neighborhood of that pixel. The principal LBP operator was given by ${ }^{40}$. The idea behind the LBP operator is to search a center

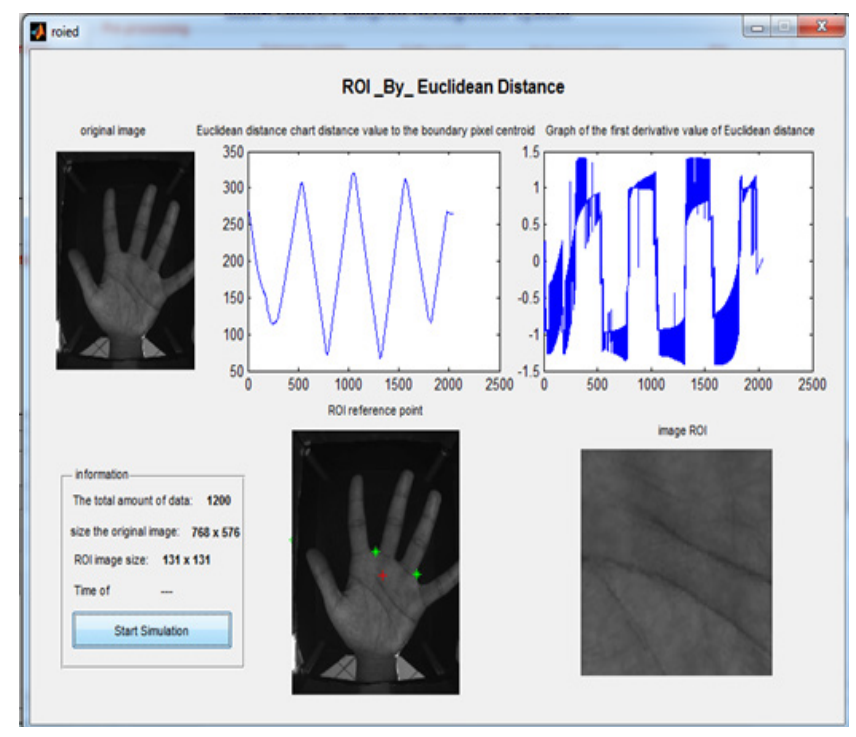

Figure 9. GUI of Automatic ROI system by using Euclidean Distance Algorithm. pixel value of an image and take this value as threshold and check as below case:

$L B P \_B i n a r y \_C o d e=\left\{\begin{array}{c}1 ; \text { if neigborhood } \\ \text { pixel }>=\text { Threshold } \\ 0 ; \text { Otherwise }\end{array}\right.$

If neighbor pixel has higher value or equal to center pixel value, the pixel takes the value 1 , otherwise it takes the value zero. The example of LBP and how to calculate a binary code is represented in Figure 10.

After that the LBP was extended to utilize neighborhoods of various sizes. In such case a circle is produced having radius $\mathrm{R}$ from the center pixel. $\mathrm{P}$ sampling points on the circle edge are determined and compared with center pixel value to find the values of all sampling points in neighborhoods for any radius and any number of pixels. Figure 11 demonstrates three neighbor sets for various values of $\mathrm{P}$ and $\mathrm{R}$.

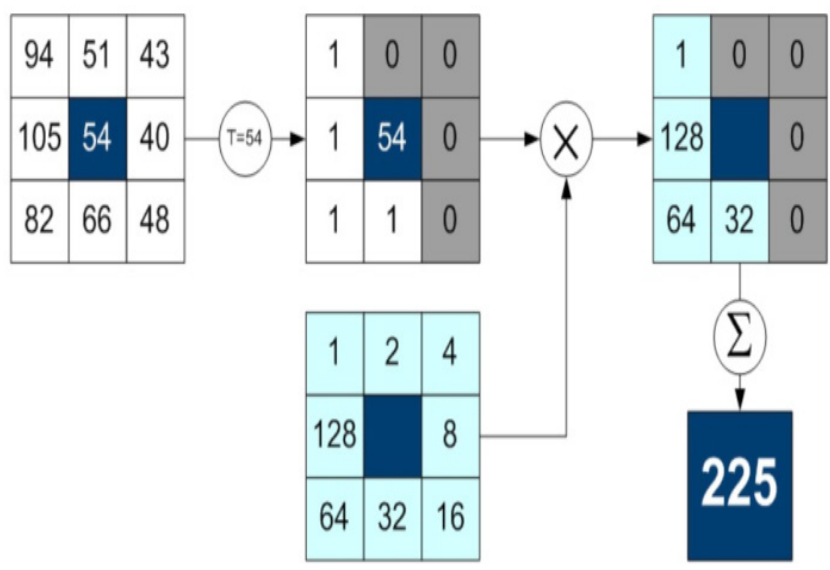

Figure 10. Stages of LBP calculation process.
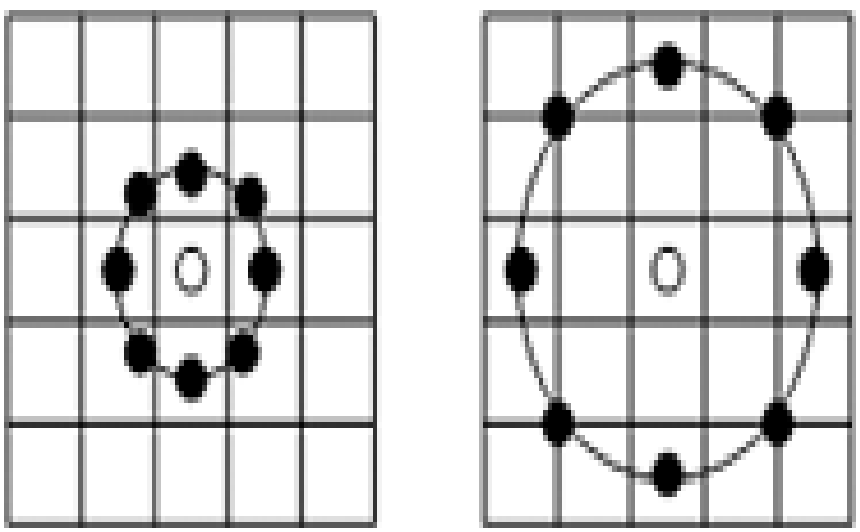

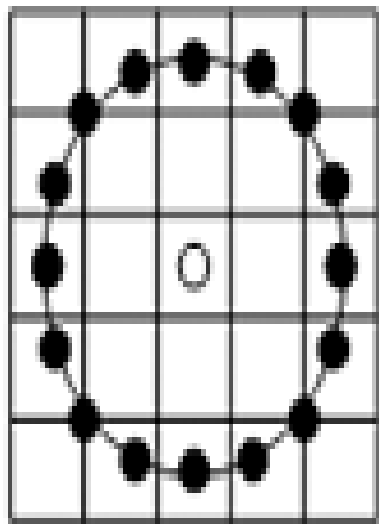

Figure 11. Circular $(8,1),(8,2)$ and $(16,2)$ neighborhoods. 


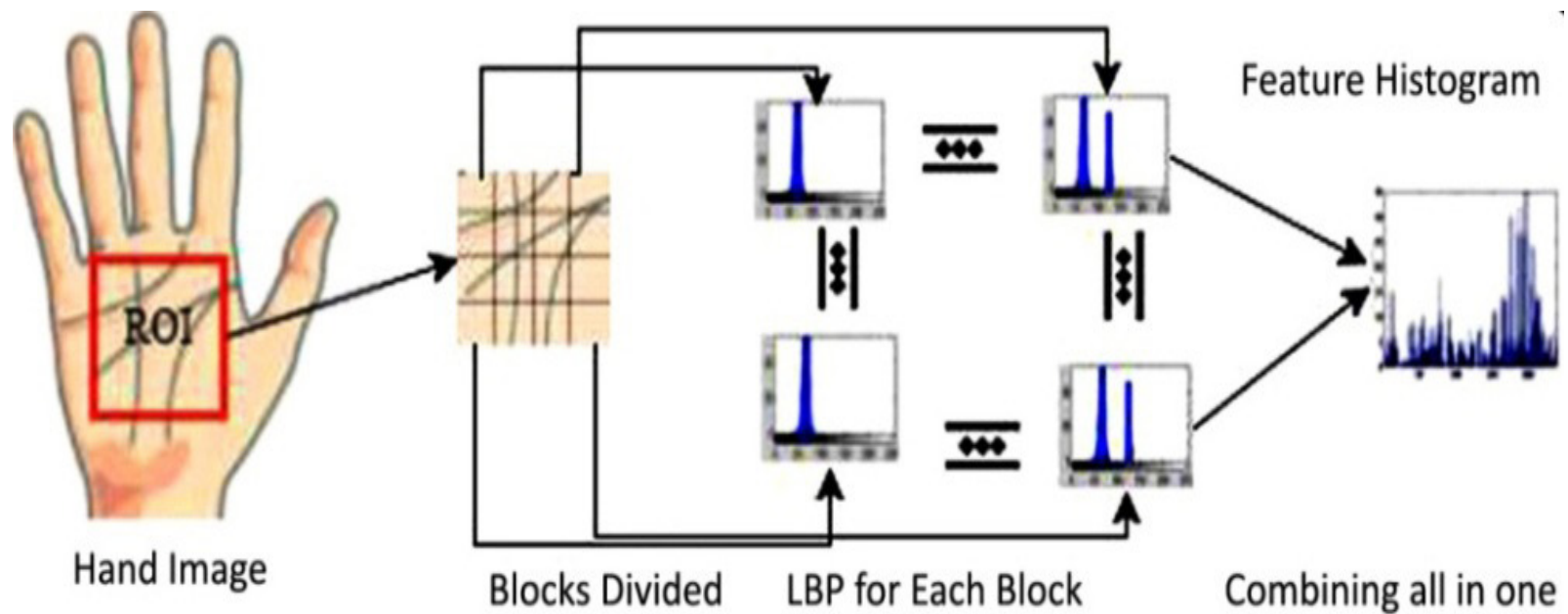

Figure 12. LBP of palmprint image with histogram of each block.

In the case of our work the LBP feature extraction was used to calculate the LBP for every pixel in the image. This occurred by divided the palmprint image into blocks or regions. Here the image was divided into $(8 \times 8)$ block size. The feature vector of the image can be generated by combining all histogram of each block. Figure 12 shows the process of our LBP technique.

Algorithm 1 describes the LBP step by step.

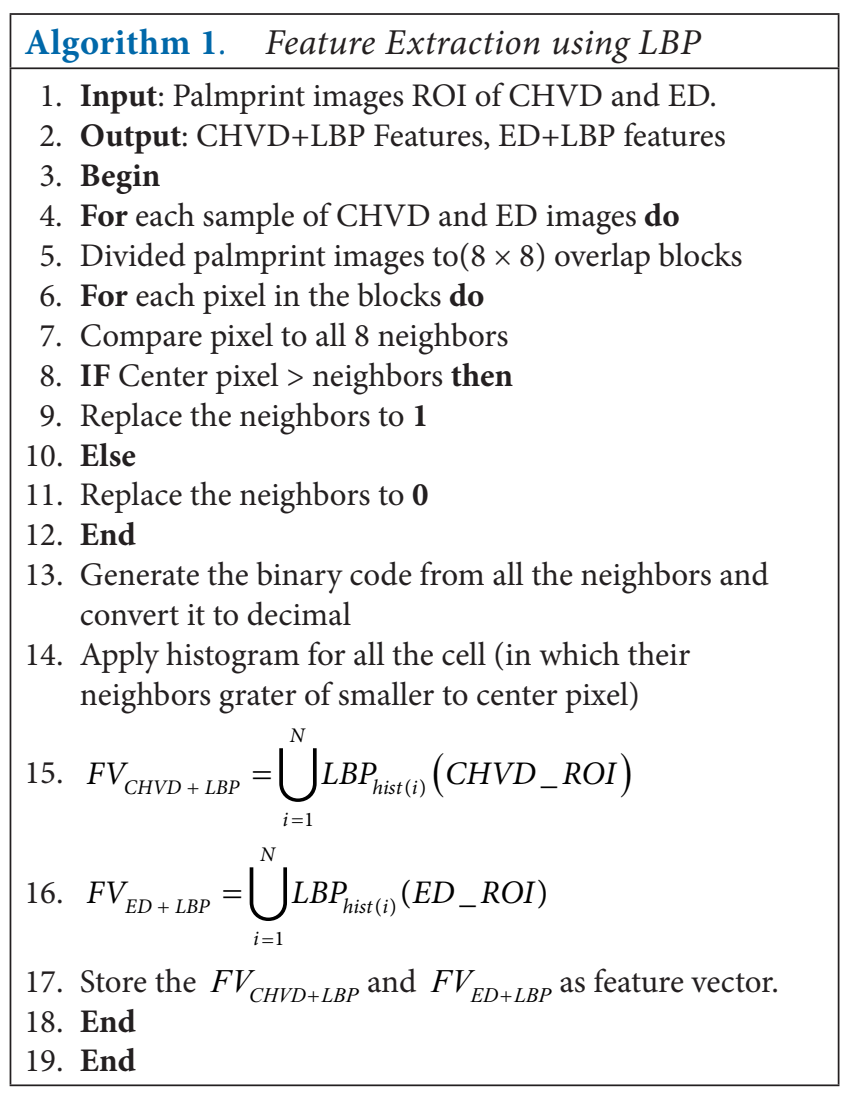

\subsection{Matching Process}

The matching was done by compared input image (Query or Test) with the template which stored in the database that taken at the time of enrollment and compute the degree of similarity or dissimilarity from two templates. To achieve the score we used Euclidean distance measure to compute the similarity or the difference between the templates, The Euclidean distance can be calculated by using Eq. (6).

$$
d\left(\text { Test }_{i}, \text { Temp }_{j}\right)=\sqrt{\sum_{i \& j=1}^{N}\left(\text { Test }_{-} V_{i}-\text { Template }_{-} V_{j}\right)^{2}}
$$

Where, $\mathrm{N}$ is the number of feature in Test $V_{-} V_{i}$ and Template_ $V_{j}$.

Algorithm 2 describes the Matching step by step.

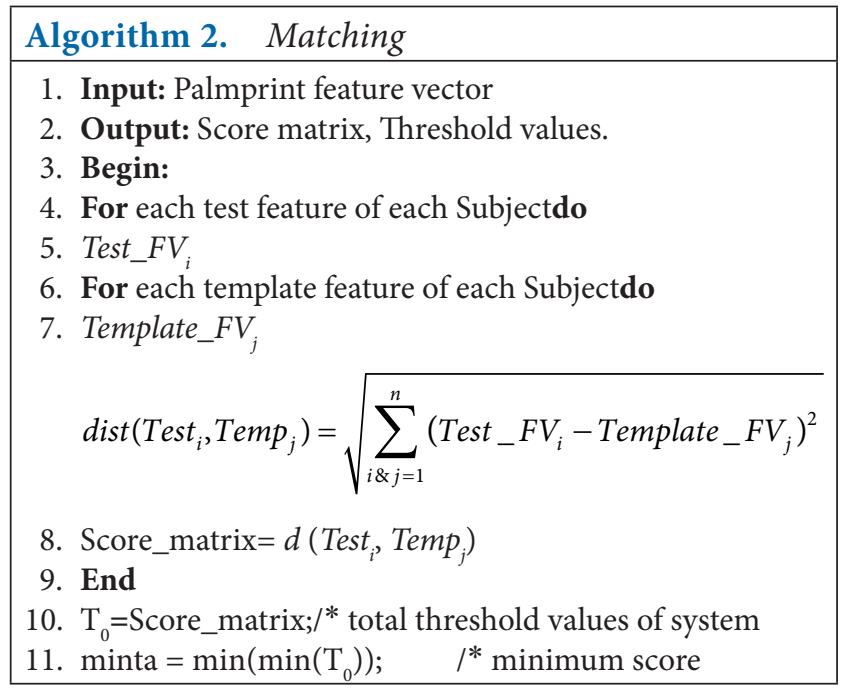




\begin{tabular}{|c|c|}
\hline 12. $\operatorname{maxta}=\max \left(\max \left(\mathrm{T}_{0}\right)\right)$; & $I^{*}$ maximum score \\
\hline 13. $\beta=100$ & $\begin{array}{l}\text { /* size of optimal threshold } \\
\text { values }\end{array}$ \\
\hline $\begin{array}{l}\text { 14. } \Delta=(\text { maxta }- \text { minta }) / \beta \text {; } \\
\text { 15. } \text { const }=1: 1: \beta \text {; } \\
\text { 16. } \text { End }\end{array}$ & $I^{*}$ threshold vector \\
\hline \multicolumn{2}{|c|}{$\begin{array}{l}\text { 17. Store score_matrix in database as math file for } \\
\text { discussion purposed. }\end{array}$} \\
\hline 18. End & \\
\hline
\end{tabular}

\subsection{Decision}

In the final steps of the study the decision was taken either "Accepted "or "Rejected "with the help of threshold value $(\mathrm{T})$. In the case of "Accepted", the distance (score $S$ ) should be $(S \leq T)$ otherwise it means "Rejected".

\section{Results and Discussion}

This section divided into different subsections. In section 3.1 dataset details are covered. The evaluation matrix is addressing in section 3.2, the results of extracting ROI by CHVD with LBP feature extraction is discussed in section 3.3. The section 3.4 addresses the results of ROI by $\mathrm{ED}$ and feature extraction techniques by using also LBP. The experimental applied on the laptop Dell, Intel core i3, CPU $2.20 \mathrm{GHz}$ with RAM 8.00GB on 64-bit operating system (windows 7) and by MATLAB software 2013a.

\subsection{Database}

The experiments were evaluated on Chinese Academy of Sciences' Institute of Automation (CASIA) ${ }^{41,42}$ MultiSpectral Database v1.0 palmprint database which has 8 bits gray level (JPEG file), image size $(768 \times 576)$. This database contains 7200 images for 100 subjects for both left and right hands. For evaluate the algorithm the 100 subjects were selected for left hand with ID 001-100 and each subjects has 6 samples each labeled 01-06 and the total dataset containing 1200 images. The experimental applied on the laptop Dell, Intel core i3, CPU $2.20 \mathrm{GHz}$ with RAM 8.00GB on 64-bit operating system (windows 7). Figure 13 shows some samples taken from CASIA dataset and their ROI part.

\subsection{Evaluation Matrix}

To evaluate any biometric system related to specific application there are different parameters namely False Accepted Rate (FAR), False Rejected Rate (FRR) and Equal Error Rate (EER). These parameters should be achieved the lowest values to get the best performance of the system. The FAR is the ratio of imposter score exceeding the threshold values divided by all the imposter score generated by the system and calculated by Eq. (7).

$$
F A R=\frac{\text { Impostor Score exceeding thershold }}{\text { All Impostor Score }} \times 100
$$

The FRR is the ratio of genuine score falling below the threshold value divided by all the genuine score generated by the system and calculated by Eq. (8).

$$
F R R=\frac{\text { Genuine Scores falling below thershold }}{\text { All Genuine Scores }} \times 100
$$

The EER can be calculated according to the Eq. (9)

$$
E E R=\frac{F A R+F R R}{2}
$$

In addition to the above parameters there is Genuine Accept Rate (GAR) which shows the relation between FAR and FRR with the help of threshold values. Also the Receiver Operating Characteristic (ROC) curves shows the FAR values which are changed related to GAR values and it shows the performance of the system. The GAR value is calculated by Eq. (10).

$$
G A R=1-F R R
$$
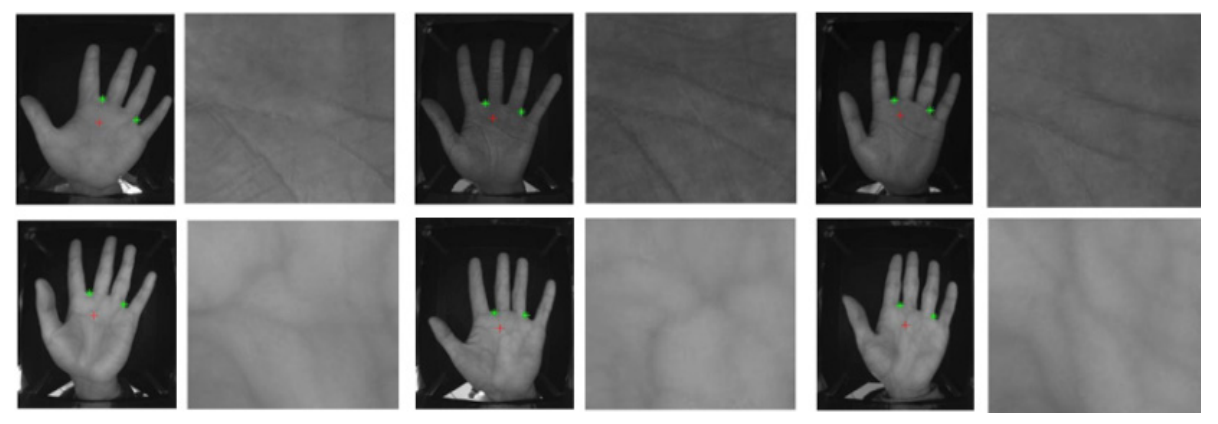

Figure 13. Samples taken from CASIA dataset with extract ROI. 


\subsection{Results of CHVD ROI with LBP}

The performance of the system was evaluated by CHVD method with LBP feature extraction technique. The size of ROI was $(155 \times 155)$ which applied on dataset size of 100 users, each user has 6 samples. The threshold values were generated from the score matrix at the matching stages. There are 100 threshold values ranged from 0 to 1 .

Table 1. Performance of the system based on CHVD with LBP Feature

\begin{tabular}{|c|c|c|c|c|}
\hline \multicolumn{5}{|c|}{ CHVD + LBP } \\
\hline T & FAR (\%) & FRR (\%) & EER (\%) & GAR (\%) \\
\hline 0 & 0 & 100 & 50 & 50 \\
\hline 0.1 & 0.035714 & 86.18182 & 43.10877 & 56.89123 \\
\hline 0.2 & 0.071429 & 62.44444 & 31.25794 & 68.74206 \\
\hline 0.3 & 0.964286 & 36.26263 & 18.61346 & 81.38654 \\
\hline 0.4 & 2.214286 & 17.83838 & 10.02633 & 89.97367 \\
\hline 0.5 & 3.464286 & 8 & 5.732143 & 94.26786 \\
\hline $\mathbf{0 . 6}$ & $\mathbf{5 . 1 4 2 8 5 7}$ & $\mathbf{2 . 6 4 6 4 6 5}$ & $\mathbf{3 . 8 9 4 6 6 1}$ & $\mathbf{9 6 . 1 0 5 3 4}$ \\
\hline 0.7 & 9.428571 & 0.707071 & 5.067821 & 94.93218 \\
\hline 0.8 & 13.42857 & 0.242424 & 6.835498 & 93.1645 \\
\hline 0.9 & 19.39286 & 0.10101 & 9.746934 & 90.25307 \\
\hline 1 & 26.60714 & 0.040404 & 13.32377 & 86.67623 \\
\hline
\end{tabular}

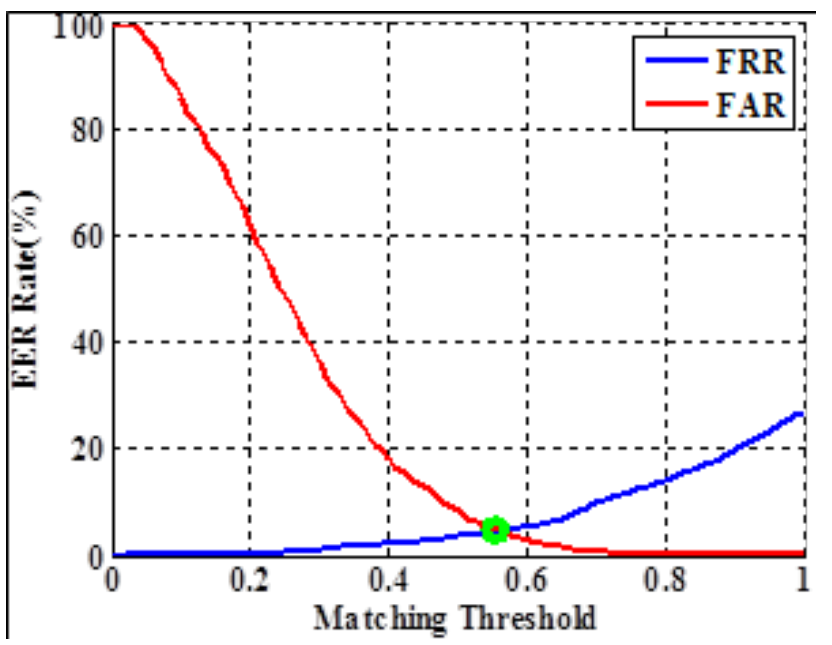

(a)
The system can achieved the best performance with minimum EER and maximum GAR which determined with help of threshold values. The system achieved different results on different threshold values e.g. on $\mathrm{T}=0$ both EER and GAR of the system were $50 \%$ that means higher EER and minimum GAR of the system. The system achieved the best performance with minimum EER of $3.894661 \%$ and maximum GAR of $96.10534 \%$ at the threshold value of 0.6 . Table 1 shows the results of the system by different $\mathrm{T}$ values with FAR, FRR, EER and GAR. Figure 14a depicts the relation between FAR and FRR with the help of threshold values and Figure 14b shows the ROC of the system by GAR Vs. FAR on different threshold values.

\subsection{Results of ED with LBP}

In the second experimental, the ED method was used to extract the ROI of $(131 \times 131)$ size then passed to LBP feature extraction method which evaluated by the same dataset applied in previous experiment. The system was achieved different results on different $T$ values reached to 100 and ranged from 0 to 1 which increased by 0.01 in each iteration and these threshold values were used to check the performance of the system. The efficiency result was achieved by minimum EER of

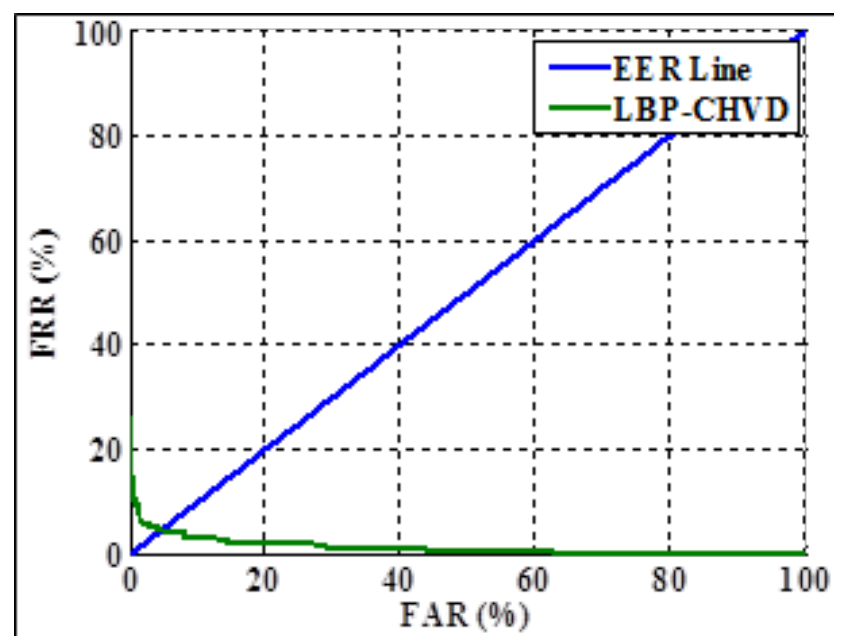

(b)

Figure 14. Performance of CHVD+LBP System: (a) Relation of FAR vs. FRR based on threshold value, and (b) ROC curve of system based on FAR vs. GAR. 
$11.76389 \%$ and Maximum GAR reached to $88.23611 \%$ which showed the best results compared with the previous technique. Table 2 shows the results of the system by different $T$ values with FAR, FRR, EER and GAR and (Figure 15a) depicts the relation between FAR and FRR with help of threshold values and (Figure 15b) shows the ROC of the system by GARVs, FAR on different threshold values.

Finally, the comparison between both ROI methods shows that ED with LBP feature technique gave the least result with higher EER reached to $11.76389 \%$ and GAR of $88.23611 \%$ while CHVD with LBP achieved the best result with minimum EER of $3.894661 \%$ and maximum GAR of $96.10534 \%$. Thecomparison between both methods based on EER and GAR is shown in (Figure 16a) and (Figure 16b) respectively.

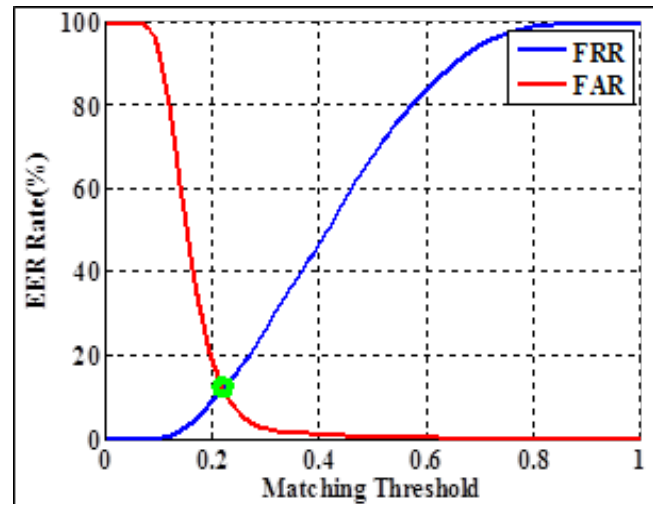

(a)
Table 2. Performance of the system based on ED with LBP feature

\begin{tabular}{|c|c|c|c|c|}
\hline \multicolumn{5}{|c|}{ ED + LBP } \\
\hline $\mathbf{T}$ & FAR (\%) & FRR (\%) & EER (\%) & GAR (\%) \\
\hline 0 & 0 & 100 & 50 & 50 \\
\hline 0.1 & 0.178571 & 95.0101 & 47.59434 & 52.40566 \\
\hline 0.2 & 9.25 & 18.0202 & 13.6351 & 86.3649 \\
\hline $\mathbf{0 . 2 2}$ & $\mathbf{1 1 . 7 5}$ & $\mathbf{1 1 . 7 7 7 7 8}$ & $\mathbf{1 1 . 7 6 3 8 9}$ & $\mathbf{8 8 . 2 3 6 1 1}$ \\
\hline 0.3 & 26 & 2.383838 & 14.19192 & 85.80808 \\
\hline 0.4 & 46.32143 & 1.111111 & 23.71627 & 76.28373 \\
\hline 0.5 & 67.82143 & 0.666667 & 34.24405 & 65.75595 \\
\hline 0.6 & 83.85714 & 0.363636 & 42.11039 & 57.88961 \\
\hline 0.7 & 94.35714 & 0.20202 & 47.27958 & 52.72042 \\
\hline 0.8 & 99 & 0.060606 & 49.5303 & 50.4697 \\
\hline 0.9 & 99.96429 & 0 & 49.98214 & 50.01786 \\
\hline 1 & 100 & 0 & 50 & 50 \\
\hline
\end{tabular}

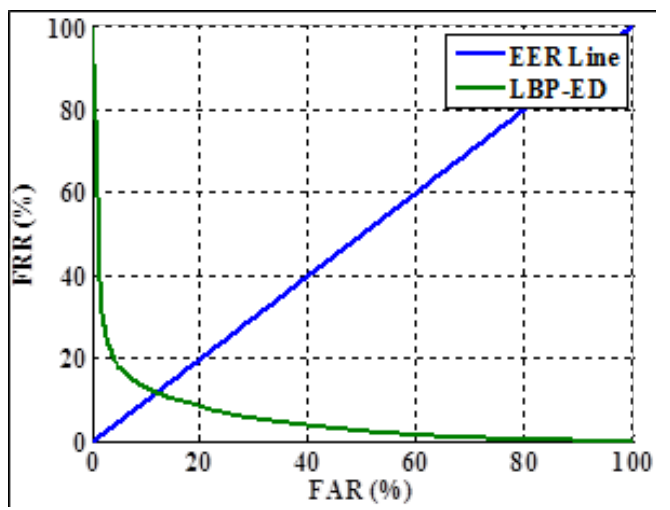

(b)

Figure 15. Performance of ED+LBP: (a) Relation of FAR vs. FRR based on threshold value, and (b) ROC curve of FAR vs. GAR for.

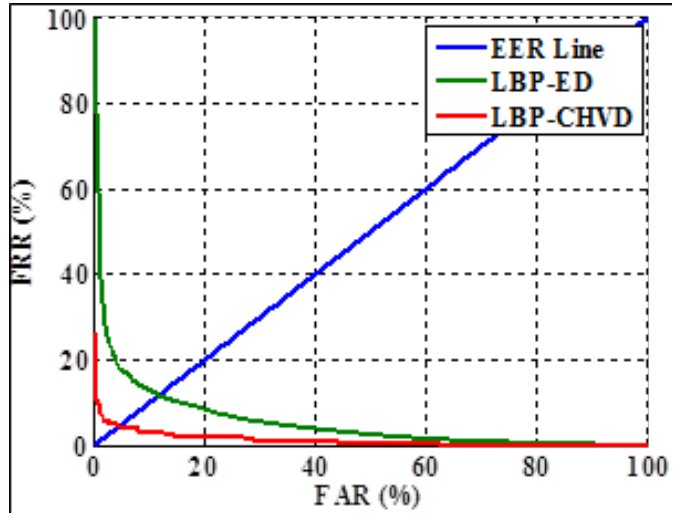

(a)

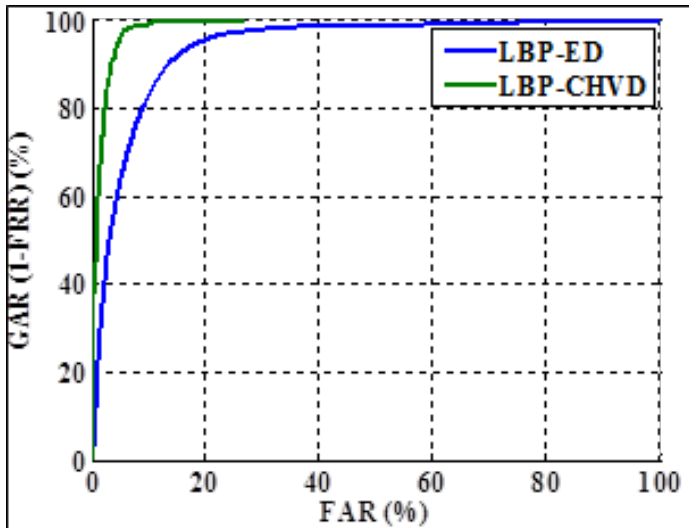

(b)

Figure 16. Comparison of GAR of CHVD and ED algorithm with LBP feature extraction: (a) EER curve, and (b) GAR Vs. FAR. 


\section{Conclusion}

This research work evaluated the performance of two ROI techniques namely CHVD and ED on CASIA MultiSpectral Database v1.0. The extraction of ROI is very important step in palmprint recognition system. It helps in extraction of features in terms of real part which contains rich information required for an authentication system. The research work evaluated ROI extracted by using CHVD and ED techniques, build feature matrix using LBP technique and PCA method which used for feature reduction. The LBP was applied for both ROIs and it was concluded from the experimental work that CHVD method achieved better performance with GAR reached to $96.10534 \%$ with EER equal to $3.894661 \%$, while the EDROI method applied to LBP and PCA gave GAR reached to $88.23611 \%$ with EER equal to $11.76389 \%$. This work may be extended to combine LBP with LPP features with neural network to achieve the best performance of the system.

\section{References}

1. Jain A, Bolle R, Pankanti R. Biometrics: Personal Identification in Networked Society. Norwell, Massachusetts: Kluwer Academic Publishers; 1999. p. 1-411. Crossref, Crossref.

2. Anil KJ, Flynn P, Ross AA. Handbook of biometrics. Springer, USA; 2007. p. 1-566.

3. NEC Automated Palmprint Identification System. Date accessed: 01/012000. https://www.policemag.com/channel/ technology/products/2000/01/automated-palmprint-identification-system.aspx.

4. Duta N, Jain AK, Mardia KV. Matching of palmprints, Pattern Recognition Letters. 2002; 23(4):477-85. Crossref.

5. Shu W, Zhang D. Automated personal identification by palmprint, Optical Engineering. 1998; 38(8):2359-62. Crossref.

6. Li F, Leung MKH, Yu X. Palmprint identification using Hausdorff distance. In: Proceedings of IEEE International Workshop on Biomedical Circuits and Systems; 2004. p. S3/3-S5-8. PMid:15336835.

7. Wu X, Wang K, Zhang D. Line feature extraction and matching in palmprint. In: Proceeding of the Second International Conference on Image and Graphics; 2002. p. 583-90. Crossref.

8. Wu X, Wang K, Zhang D. Fuzzy direction element energy feature (FDEEF) based palmprint identification, Object Recognition Supported by User Interaction for Service Robots. 2002; 1:95-98.
9. Boles WW, Chu SYT. Personal identification using images of the human palms. In: Proceedings of IEEE Region 10 Annual Conference, Speech and Image Technologies for Computing and Telecommunications; 1997. 1, p. 295-98. Crossref.

10. Rafael Diaz M, Travieso CM, Alonso JB, Ferrer MA. Biometric system based in the feature of hand palm. In: Proceedings of $38^{\text {th }}$ Annual International Carnahan Conference on Security Technology; 2004. p. 136-39.

11. Kung SY, Lin SH, Fang M. A neural network approach to face/palm recognition. In: Proceedings of IEEE Workshop Neural Networks for Signal Processing; 1995. p. 323-32. Crossref.

12. Rodrigues BAR, Silva JDL. Biometric identification by dermatoglyphics. In: Proceedings of International Conference on Image Processing; 1996. 1, p. 319-22.

13. Wu W, Wang K, Zhang D. A novel approach of palmline extraction. In: Proceeding of the Third International Conference on Image and Graphics; 2004. p. 230-33. PMid:15193533.

14. Ali MHM, Yannawar P, Gaikwad AT. Study of edge detection methods based on palmprint lines. In: Proceedings of International Conference on Electrical, Electronics, and Optimization Techniques (ICEEOT). Chennai, India; 2016. p. 1344-50. Crossref.

15. Connie T, Jin ATB, Ong MGK, Ling DNC. An automated palmprint recognition system, Image and Vision Computing. 2005 May; 23(5):501-15. Crossref.

16. Wu X, Zhang D, Wang K. Fisherpalms based palmprint recognition, Pattern Recognition Letters. 2003 Nov; 24(15):2829-38. Crossref.

17. Lu G, Zhang D, Wang K. Palmprint recognition using Eigenpalms features, Pattern Recognition Letters. 2003 Jun; 24(9-10):1463-67. Crossref.

18. Jing XY, Zhang D. A face and palmprint recognition approach based on discriminant DCT feature extraction, IEEE Transactions on Systems, Man, and Cybernetics, Part B (Cybernetics). 2004 Dec; 34(6):2405-15. Crossref.

19. Li Q, Qiu Z, Sun D. Feature-level fusion of hand biometrics for personal verification based on Kernel PCA. International Conference on Biometrics. Springer, Berlin; 2006. p. 744-50.

20. Lu GM, Wang KQ, Zhang D. Wavelet based independent component analysis for palmprint identification. In: Proceedings of International Conference on Machine Learning and Cybernetics; 2004 Aug. 6, p. 3547-50. Crossref.

21. Chu R, Lei Z, Han Y, Li SZ. Learning Gabor magnitude features for palmprint recognition. In Asian Conference on Computer Vision ACCV. Springer, Berlin, Heidelberg; 2007. p. 22-31. Crossref. 
22. Ekinci M, Aykut M. Palmprint recognition by applying wavelet sub and representation and kernel PCA. In International Workshop on Machine Learning and Data Mining in Pattern Recognition. Springer, Berlin, Heidelberg; 2007 Jul. p. 628-42. Crossref, Crossref.

23. Han CC, Cheng HL, Lin CL, Fan KC. Personal authentication using palm-print features, Pattern Recognition. 2003; 36(2):371-81. Crossref.

24. You J, Kong WK, Zhang D, Cheung KH. On hierarchical palmprint coding with multiple features for personal identification in large databases, IEEE Transactions on Circuits and Systems for Video Technology. 2004; 14(2):234-43. Crossref.

25. Lu G, Wang K, Zhang D. Wavelet based feature extraction for palmprint identification. In: Proceeding of Second International Conference on Image and Graphics; 2002. p. 780-85. Crossref.

26. Wu X, Wang K. Zhang D. Palmprint recognition using directional energy feature. In: Proceedings of International Conference on Pattern Recognition; 2004. 4, p. 475-78.

27. Mouad MHA, Gaikwad AT. Multimodal Biometrics Enhancement Recognition System based on Fusion of Fingerprint and PalmPrint: A Review, Global Journal of Computer Science and Technology. 2016 Sep; 16(2):13-26.

28. Poon C, Wong DCM, Shen HC. Personal identification and verification: fusion of palmprint representations. In: Proceedings of International Conference on Biometric Authentication, Springer, Berlin, and Heidelberg; 2004. p. 782-88. Crossref.

29. Kumar A, Shen HC. Palmprint identification using PalmCodes. In: Proceedings of 3rd International Conference on Image and Graphics; 2004. p. 258-61. Crossref.

30. Mouad MHA, Yannawar PL, Gaikwad AT. Multi-Algorithm of Palmprint Recognition System Based on Fusion of Local Binary Pattern and Two-Dimensional Locality Preserving Projection, Procedia Computer Science. 2017 Dec; 115:482-92. Crossref.

31. Wang X, Gong H, Zhang H, Li B, Zhuang Z. Palmprint identification using boosting local binary pattern. In: Proceedings of International Conference on Pattern Recognition; 2006 Aug. 3, p. 503-06.
32. Pang $\mathrm{YH}$, Connie T, Jin A, Ling D. Palmprint authentication with Zernike moment invariants. Proceedings of the $3^{\text {rd }}$ IEEE International Symposium on Signal Processing and Information Technology; 2003 Dec. p. 199-202.

33. Zhang L, Zhang D. Characterization of palmprints by wavelet signatures via directional context modeling, IEEE Transactions on Systems, Man and Cybernetics. 2004 Jun; 34(3):1335-47. Crossref.

34. Dai Q, Bi N, Huang D, Zhang D, Li F. M-band wavelets applications to palmprint recognition based on texture features. In: Proceedings Conference on Image Processing; 2004 Oct. 2, p. 893-96.

35. Li Y, Wang K, Zhang D. Palmprint recognition based on translation invariant Zernike moments and modular neural network. International Symposium on Neural Networks; 2005 May. p. 177-82. Crossref.

36. Noh JS, Rhee KH. Palmprint identification algorithm using $\mathrm{Hu}$ invariant moments and Otsu binarization. In: Proceeding of Fourth Annual ACIS International Conference on Computer and Information Science; 2005. p. 94-99.

37. Zhang DD. Palmprint authentication. Springer Science and Business Media; 2004. p. 1-241.

38. Wirayuda TAB, Habbi AA, Didik HK, Retno ND. Real-time hand-tracking on video image based on palm geometry. In Information and Communication Technology (ICoICT), International Conference; 2013 Mar. p. 241-46. Crossref.

39. Xin P, Qiu-Qi R. Palmprint recognition using Gabor feature-based (2D) 2 PCA, Neurocomputing. 2008 Aug; 71(13):3032-36.

40. Timo O, Pietikäinen M, Harwood D. A comparative study of texture measures with classification based on featured distributions, Pattern recognition. 1996 Jan; 29(1):51-59. Crossref.

41. Biometrics Ideal Test. Fingerprint Image Database Version 5.0. Date accessed: 2014. http://biometrics.idealtest.org/.

42. Hao Y, Sun Z, Tan T, Ren C. Multi-spectral palm image fusion for accurate contact-free palmprint recognition. Proceedings of IEEE International Conference on Image Processing; 2008 Oct. p. 281-84. 